\title{
Combined ChIP-Seq and transcriptome analysis identifies AP-1/JunD as a primary regulator of oxidative stress and IL-1 $\beta$ synthesis in macrophages
}

\author{
Richard P Hull', Prashant K Srivastava' ', Zelpha D'Souza', Santosh S Atanur ${ }^{1}$, Fatima Mechta-Grigoriou², \\ Laurence Game ${ }^{1}$, Enrico Petretto ${ }^{1}, \mathrm{H}$ Terence Cook ${ }^{3}$, Timothy J Aitman ${ }^{1}$ and Jacques Behmoaras ${ }^{3 *}$
}

\begin{abstract}
Background: The oxidative burst is one of the major antimicrobial mechanisms adopted by macrophages. The WKY rat strain is uniquely susceptible to experimentally induced macrophage-dependent crescentic glomerulonephritis (Crgn). We previously identified the AP-1 transcription factor JunD as a determinant of macrophage activation in WKY bone marrow-derived macrophages (BMDMs). JunD is over-expressed in WKY BMDMs and its silencing reduces Fc receptor-mediated oxidative burst in these cells.

Results: Here we combined Jund RNA interference with microarray analyses alongside ChIP-sequencing (ChIP-Seq) analyses in WKY BMDMs to investigate JunD-mediated control of macrophage activation in basal and lipopolysaccharide (LPS) stimulated cells. Microarray analysis following Jund silencing showed that Jund activates and represses gene expression with marked differential expression ( $>3$ fold) for genes linked with oxidative stress and IL-1 $\beta$ expression. These results were complemented by comparing whole genome expression in WKY BMDMs with Jund congenic strain (WKY.LCrgn2) BMDMs which express lower levels of JunD. ChIP-Seq analyses demonstrated that the increased expression of JunD resulted in an increased number of binding events in WKY BMDMs compared to WKY.LCrgn2 BMDMs. Combined ChIP-Seq and microarray analysis revealed a set of primary JunD-targets through which JunD exerts its effect on oxidative stress and IL-1 $\beta$ synthesis in basal and LPS-stimulated macrophages.

Conclusions: These findings demonstrate how genetically determined levels of a transcription factor affect its binding sites in primary cells and identify JunD as a key regulator of oxidative stress and IL-1 $\beta$ synthesis in primary macrophages, which may play a role in susceptibility to Crgn.
\end{abstract}

\section{Background}

Macrophages are efficient phagocytes of the immune system that produce reactive oxygen species (ROS) during the phagocytosis of pathogens, considered as a marker of cell activation. The well-established classical pathway of macrophage activation induced by interferon (IFN) $-\gamma$ and/or lipopolysaccharide (LPS) is known to play a vital role in host defence during inflammation. Macrophages activated in this manner express high

\footnotetext{
* Correspondence: jacques.behmoaras@imperial.ac.uk

${ }^{3}$ Centre of Complement and Inflammation Research, Imperial College

London, Du Cane Road W12 ONN, London, UK

Full list of author information is available at the end of the article
}

levels of proinflammatory cytokines and reactive oxygen and nitrogen intermediates that are crucial in the defence against intracellular pathogens [1,2]. The AP-1 transcription factor plays a key role in regulating cell growth and environmental stress responses [3-5]. In classically activated (M1) macrophages, AP-1 plays a central role together with NF- $\mathrm{kB}$ in signal-dependant gene expression that is crucial for innate immunity [6]. JunD is a member of AP-1 that is constitutively expressed and has been previously shown to protect cells from oxidative stress and to reduce tumour angiogenesis by limiting the production of ROS [7]. The chronic oxidative stress generated by the inactivation of JunD, has

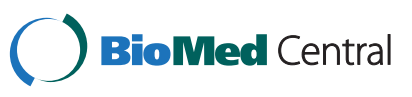


been shown to promote aging and increase tumour development $[8,9]$. In various tissues, including the kidney, the absence of JunD led to the over-expression of hypoxia inducible factor (HIF)-target genes in podocytes, most likely as a result of increased oxidative stress [10].

Wistar Kyoto (WKY) rats are uniquely susceptible to nephrotoxic nephritis (NTN), a rat model of crescentic glomerulonephritis (Crgn) [11]. The macrophages of this strain show a 20-fold increase in Jund mRNA expression as well as increased specific JunD protein binding to AP1 consensus sequence nucleotides ( $5^{\prime}$-TGAGTCA-3 ${ }^{\prime}$ ) when compared with the NTN-resistant LEW strain [12]. In addition WKY BMDMs show greater superoxide anion production when stimulated with PMA (unpublished observations) and significantly increased NOS2 expression [13] when stimulated with LPS, suggesting that the macrophages of this strain have a genetically determined pro-inflammatory phenotype characterised by increased oxidative stress. We have previously shown that JunD is a determinant of the macrophage oxidative burst associated with crescentic glomerulonephritis. In a genome-wide linkage analysis and haplotype analysis for NTN-related phenotypes in WKY and LEW rats, we delineated a minimal genomic region of $130 \mathrm{~kb}$ on rat chromosome 16 where Jund was the only markedly over-expressed transcript. The functional role of JunD was established by siRNA knock-down of Jund in WKY BMDMs [12] which resulted in reduced Fc receptor mediated oxidative burst confirming the previously reported antioxidant role of JunD in other tissues [7,9]. Furthermore, the role of JunD in TLR4-induced primary human macrophage activation was established. siRNA knockdown of JUND in these cells resulted in a significantly reduced secretion of TNF $\alpha$, IL-6 and IL-10 [12]. One possible mechanism for this was suggested by Smolinska and colleagues who showed that Hck kinase mediates TLR4-induced transcription of both TNF and IL-6 through binding of AP-1 heterodimers composed of cFos and JunD [14]. Based on these results, we hypothesised that JunD controls respiratory burst and the related oxidative stress in basal and classically activated (LPS/ TLR4, M1) macrophages.

To identify genes and pathways regulated by JunDmediated macrophage activation in WKY BMDMs, we have carried out microarray-based gene expression studies following siRNA knock down of Jund in basal and LPS-stimulated conditions. ChIP-Seq analysis was performed on basal and LPS-stimulated WKY BMDMs and used to complement the microarray results in order to identify primary JunD targets. ChIP-Seq and microarray analyses were also carried out in a Jund congenic strain (WKY.LCrgn2) known to have reduced JunD mRNA and protein levels [12]. In this strain, the Jund locus was transferred from the Lewis strain into the WKY strain by back-crossing over nine generations. Genome-wide integration of all datasets identified primary JunD-target genes and a regulatory network involved in oxidative stress and IL-1 $\beta$ expression in macrophages leading to increases in mature IL- $1 \beta$ production in BMDMs and glomeruli from the WKY strain.

\section{Results}

Jund regulates macrophage gene expression that controls primarily oxidative stress and IL-1 $\beta$ synthesis

A description of the macrophage function related to different levels of JunD expression in different inbred rat is summarised in Table 1 . In order to identify genes under the transcriptional control of JunD in primary macrophages, expression levels of Jund were first silenced by RNA interference in the WKY BMDMs that overexpress JunD (Figure 1) [12]. Following confirmation of knockdown by qRT-PCR and Western blot (Figure 2A and $\mathrm{C}$ ), the samples were subjected to microarray analysis (Additional file 1: Figure S1A). Because AP1/JunD regulates lipopolysaccharide (LPS)-TLR4 mediated cytokine secretion in primary human macrophages [12,14], microarray analysis was also performed in samples treated with Jund siRNA (48h) and stimulated with LPS for $8 \mathrm{~h}$ (Figure 2B and Additional file 1: Figure S1B). Genome-wide analysis of the BMDM transcriptome identified 1672 differentially expressed genes between unstimulated BMDMs transfected with Jund siRNA compared to scrambled control and 1,476 differentially expressed genes following 8 hours of LPS $(100 \mathrm{ng} / \mathrm{ml})$ stimulation $(\mathrm{FDR}<5 \%)$. JunD acted both as an activator and as a repressor of gene expression in BMDMs. Transfection of WKY BMDMs with Jund siRNA, thereby lowering Jund expression, resulted in the reduced expression of 868 genes out of 1672 ( 50\%) demonstrating that Jund had an activatory role in gene transcription. Alongside this, Jund knockdown also increased the expression of 804 genes ( 50\%) demonstrating that Jund could also have a repressive effect on transcription. After eight hours of LPS stimulation, 638 genes had reduced expression in the Jund siRNA knockdown group compared to controls and 838 genes demonstrated higher expression following Jund knockdown. Validation by qRT-PCR of a set of differentially expressed genes following Jund siRNA knock-down that encompassed a range of fold change differences between the two siRNA groups confirmed the microarray findings (Figure $3 \mathrm{~A}$ and $\mathrm{B}$, Additional file 2: Table S1) in 21 out of the 23 (91\%) genes selected. The directional change (activation or repression) of the microarray data was confirmed in all 23 genes.

Amongst the genes showing the most marked expression changes following Jund siRNA knock-down in WKY BMDMs, 11 genes were found to have greater 
Table 1 JunD levels and macrophage oxidative burst in rat strains used for the combined ChIP-Seq and transcriptome approach

\begin{tabular}{ccc}
\hline Strain & JunD levels in macrophages & Macrophage activation $^{* *}$ \\
\hline WKY & +++ & +++ \\
WKY.LCrgn2 & + & + \\
Lewis & + & + \\
\hline
\end{tabular}

* Jund expression levels and JunD/AP-1 protein binding measured by qRT-PCR and TransAm assay [12].

** Macrophage activation assessed by Fc receptor mediated oxidative burst [12].

than threefold differences in expression between the two siRNA groups (scrambled vs. Jund siRNA) in the unstimulated state and 2 genes were identified in the LPS stimulated state (Table 2). Interestingly, the individual functions of the majority of these genes belong to two main categories: oxidative stress (Mt2a [15], Lcn2 [16], Vcan [17], Hspb1 [18], Prkca [19]) and IL-1 $\beta$ synthesis (Klrb1a [20], Il1b, Nlrp3 [21]). This suggests that JunD has a primary role in regulating macrophage gene expression associated with oxidative stress and IL-1 $\beta$ synthesis. To investigate this further, we performed genome-wide expression analysis by microarrays over an eight hour time course of LPS stimulation in WKY and WKY.LCrgn2 BMDMs (Additional file 1: Figure S1C). The latter is a Jund congenic strain generated by introgression of the Jund locus on chromosome 16 from the Lewis donor onto the WKY recipient genome. WKY. LCrgn2 rats have significantly less Jund mRNA and JunD protein levels in their BMDMs when compared with parental WKY BMDMs (Table 1). The microarray results between the WKY and WKY.LCrgn2 BMDMs identified that 830 genes were differentially expressed over the

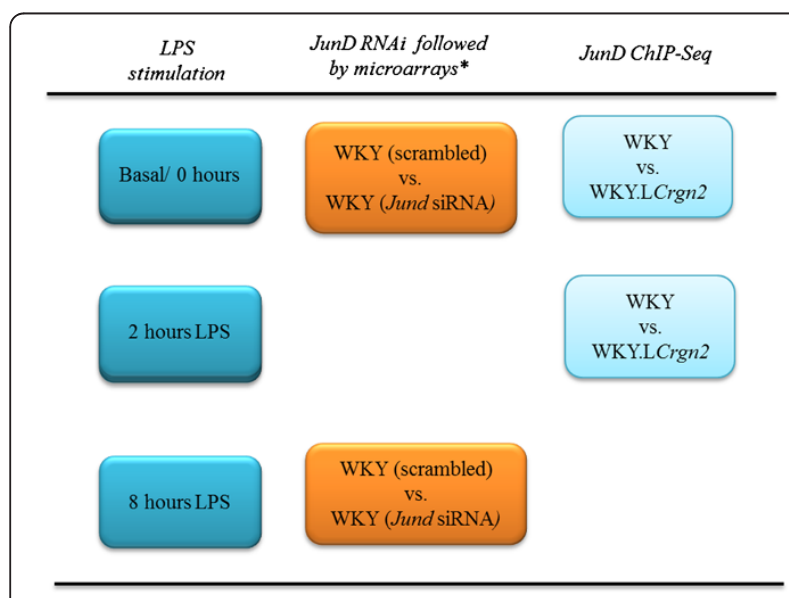

Figure 1 Strategy employed to identify primary JunD targets in bone marrow derived macrophages. We performed Jund RNAi and compared whole genome expression profiling in macrophages (basal and stimulated with LPS, 100 $\mathrm{ng} / \mathrm{ml}$, 8h) transfected with scrambled siRNA to those transfected with Jund siRNA. ChIP-Seq analysis was performed in WKY and WKY.LCrgn2 BMDMs (basal and stimulated with LPS, 100ng/ml, 2h). Transcripts showing a fold change $>3$ in the RNAi dataset were examined for JunD/AP1 peaks. eight hour timecourse. They were functionally associated with multiple immune terms focused on responses to stimuli including LPS and the regulation of cell activation (Table 3) and were validated by qRT-PCR (Additional file 1: Figure S2). A set of 201 genes that were common with the Jund siRNA knockdown was identified. Amongst these, seven transcripts showed a fold change greater than 2 (FDR $<5 \%$ ) including metallothionein 2A (Mt2a), arginase (Arg1) and cysteine dioxygenase, type 1 (Cdo1), genes associated with oxidative stress. The significant differential expression of these transcripts together with Jund between WKY and WKY.LCrgn2 BMDMs was confirmed by qRT-PCR (Figure 3C-F). Taken together the combined RNAi and congenic whole genome differential expression analysis identifies genes primarily regulating oxidative stress and IL-1 $\beta$ synthesis under transcriptional control of JunD.

\section{JunD expression levels determine the extent of the JunD cistromes}

We next combined our microarray approaches investigating JunD-mediated transcriptional control of genes with a cistrome analysis of JunD between basal and two hour LPS stimulated WKY and WKY.LCrgn2 BMDMs using ChIP-Seq (Figure 1). The aligned sequencing reads (Additional file 2: Table S2) were analysed using BayesPeak $[22,23]$ in order to identify areas of sequencing enrichment that signified JunD-binding events, termed peaks. This analysis identified a greater number of peaks in WKY basal and LPS-stimulated BMDMs compared to WKY.LCrgn2 BMDMs in both conditions (Table 4). Peaks were linked to a gene if they were located within 20 kilobases of the transcriptional start site or were located within the gene body. This meant that genetically determined differences in JunD levels resulted in an almost $50 \%$ reduction in the number of ChIP-Seq peaks that were linked to a protein coding gene in the basal state and an $87 \%$ reduction after LPS stimulation (Table 4). Fourteen peaks were successfully validated by ChIP-qPCR in WKY BMDMs with all the peaks showing at least two-fold enrichment for JunD above background (Additional file 1: Figure S3A and $\mathrm{S} 3 \mathrm{~B})$. The reduced level of JunD expression in the WKY.LCrgn2 strain was reflected in the lower levels of enrichment for the seven peaks analysed 
A

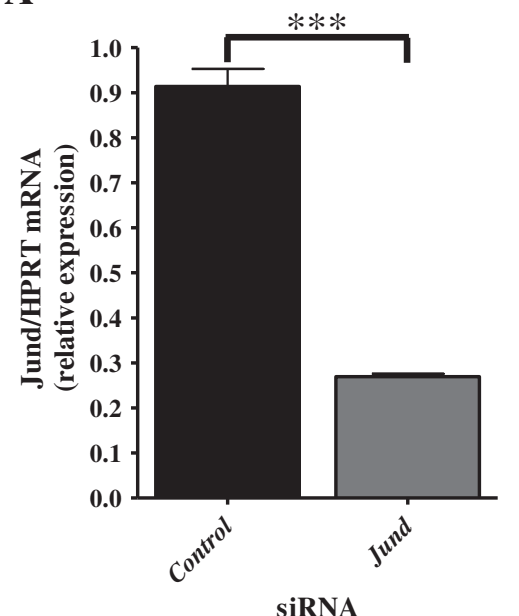

SiRNA
B

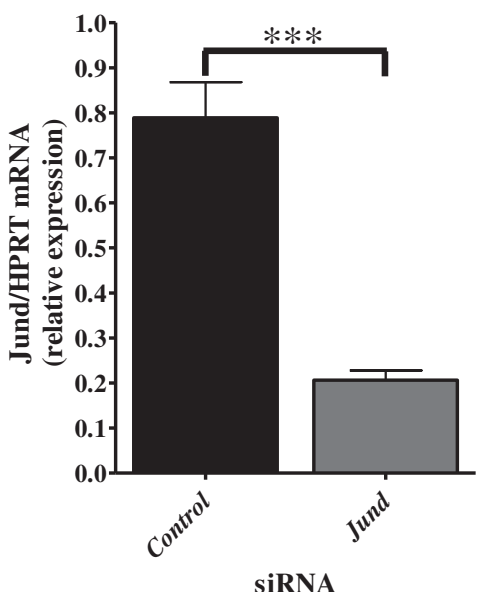

SiRNA

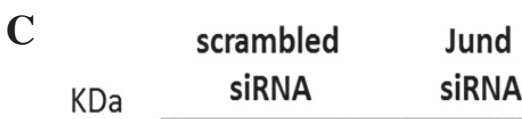

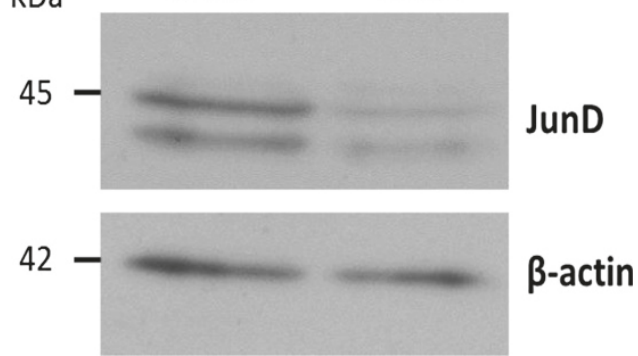

Figure 2 siRNA mediated knockdown of Jund. Assessment of the efficiency of siRNA knockdown of Jund in the unstimulated state (A, C) and following eight hours LPS stimulation (B) using qRT-PCR and Western blotting. siRNA experiments were performed in 4 different WKY rats in triplicate. ${ }^{* *} \mathrm{P}<0.001$ using two tailed unpaired t-test to compare BMDMs transfected for 48 hours with either scrambled control siRNA or Jund siRNA. The Western blot (C) for JunD is representative of four different Jund silencing experiments in WKY BMDMs and is demonstrated alongside $\beta$-actin loading control.

(Additional file 1: Figure S3C). The majority of ChIP-Seq peaks in all the strains were located in intergenic regions (35-44\% across the datasets) whilst the WKY BMDMs had a higher proportion of JunD peaks within the body of the gene (exon or intron) compared to WKY.LCrgn2 BMDMs in both the unstimulated and LPS stimulated states (Figure 4A). The distance from the nearest gene to each peak was calculated and peaks were found to be preferentially located close to the TSS in both strains (Figure 4B). De novo motif discovery using HOMER identified a 12 base pair motif in $63 \%$ of peaks in WKY LPS stimulated BMDMs with a strong similarity to the consensus AP-1 motif that has been previously recognised and shown to be functionally active [24] (Figure 4C). Additional motifs identified included CAC binding and REST/NRSF in basal WKY BMDMs (Figure 4D) and the Ascl2, CACCC binding motif for KLF factors, Eomes and Ets (Figure 4E) in LPS stimulated WKY BMDMs. Furthermore the JunD binding to the promoters of $I l 1 b$ and Prkca, two of the most markedly differentially expressed transcripts following Jund siRNA were confirmed by ChIPqPCR in both the basal and LPS stimulated conditions (Figure 4F, Additional file 1: Figure S4)

Gene ontology analysis of the JunD-bound genes in the different strains and states identified marked similarities between the enriched terms in basal WKY BMDMs (Additional file 2: Table S3) and WKY.LCrgn2 BMDMs (Additional file 2: Table S4) as well as LPS stimulated WKY.LCrgn2 BMDMs (Additional file 2: Table S5) covering multiple autoimmune disease pathways and core macrophage functions such as antigen processing. This suggested that regardless of expression level, JunD binds to genes involved in core macrophage processes. Following LPS stimulation in WKY BMDMs enrichment was seen for immune processes linked with the response of the macrophage to stimulation such as intracellular signalling cascades and the MAPK signalling pathway (Additional file 2: Table S6) highlighting the role played by JunD in the LPS response. 


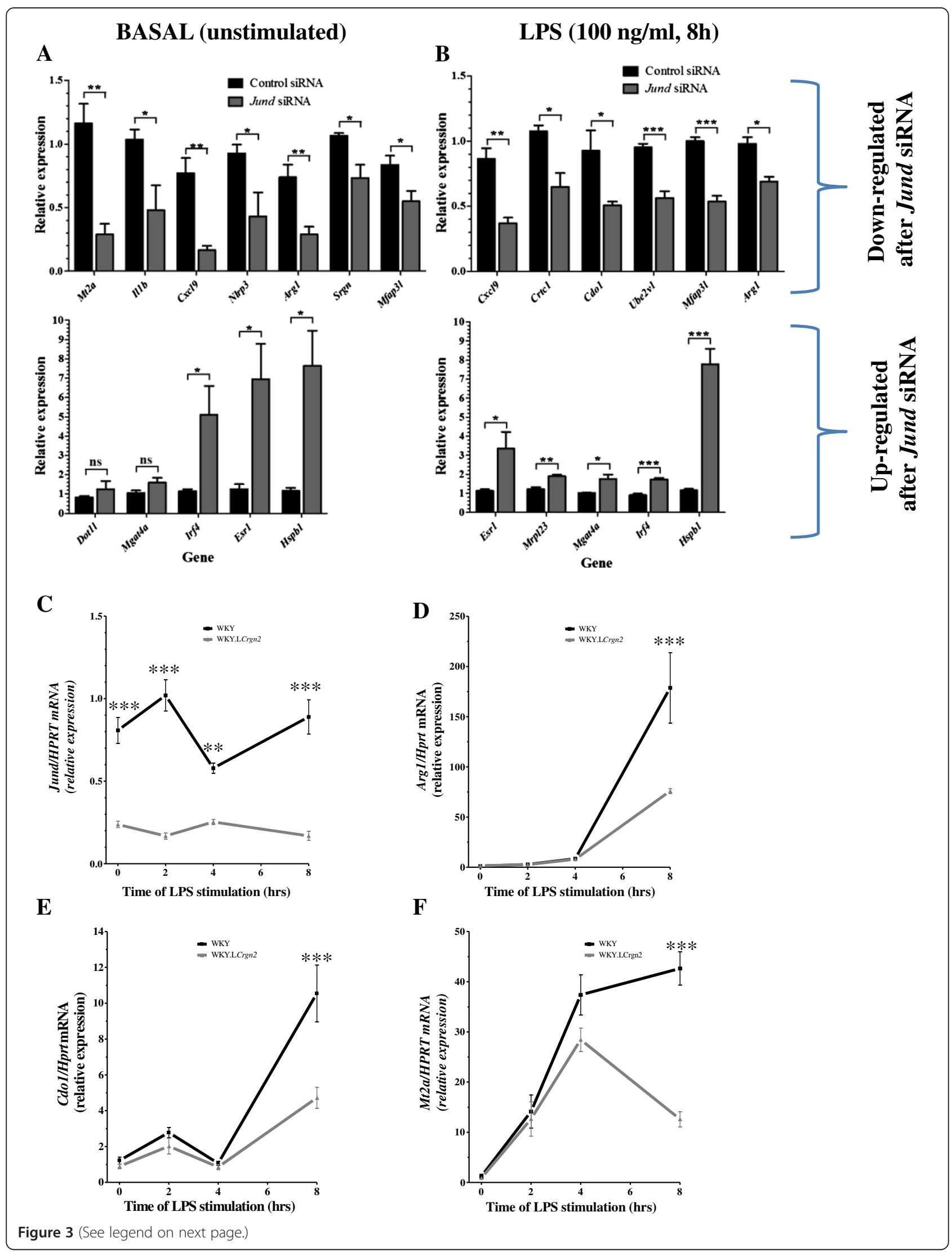


(See figure on previous page.)

Figure 3 Validation of microarray results confirms that JunD has both activatory and repressive roles in controlling gene expression linked with oxidative stress. Validation of differentially expressed genes were carried out using four biological replicates with three technical amplification replicates per siRNA type for the unstimulated (A) and eight hour LPS stimulated (B) data sets. Relative gene expression was normalised to Hprt and used to generate fold change values. ${ }^{*} \mathrm{P}<0.05 ;{ }^{*} \mathrm{P}<0.01 ;{ }^{*}{ }^{*} \mathrm{P}<0.001$ using a two-tailed unpaired t-test to detect statistically significant differences between the siRNA groups. Error bars represent standard error of the mean. Confirmation of the differential expression of Jund between WKY and WKY.LCrgn2 BMDMs (C) and key JunD targets; Arg1 (D), Cdo1 (E) and Mt2a (F), influenced by Jund siRNA knockdown with $>2$ fold change in expression that were also differentially expressed between WKY and WKY.LCrgn2 BMDMs over a timecourse of LPS stimulation. Samples from the WKY and WKY.LCrgn2 strains were amplified using a set of four biological replicates with three technical replicates per sample. ${ }^{* *} \mathrm{P}<0.001$ statistically significantly different to WKY using a two way ANOVA to compare the overall timecourse with Bonferonni's post-tests to compare individual time points.

Integration of microarray and ChIP-Seq datasets identifies primary JunD targets in macrophages

In order to identify the set of primary JunD targets, we integrated all the genomic datasets and identified the genes that correlated closely with the expression pattern of Jund during the LPS timecourse that were also differentially expressed following siRNA knockdown of Jund and showed a ChIP-Seq peak in WKY BMDMs. This identified two major networks (basal and LPS) with 24 genes in the basal state (Figure 5, upper panel) and 36 genes after LPS stimulation (Figure 5, lower panel) as primary JunD targets through which JunD mediates its effect on macrophage activation. The primary JunD targets correlate with Jund expression levels in two independent datasets (microarray analysis on WKY and WKY.LCrgn2 BMDM LPS time course and microarray on Jund siRNA in WKY BMDMs) and have a JunD ChIP-Seq peak in WKY BMDMs. These are genes where the expression is under direct control of JunD/AP1 binding, correlating with cellular JunD levels. The transcription factor Runx1 was identified as primary Jund targets together with several genes involved in oxidative stress such as Trpv4, Vav2, Ifi30, Nqo2, and P2ry2, Ctnnb1 and Bcl2l1 Additional file 1: Figure S5).

JunD expression levels determine active IL-1 $\beta$ secretion in primary macrophages and nephritic glomeruli

Since the previous role of JunD is regulating macrophage oxidative burst is known [12] and our data shows that JunD regulates genes involved in IL-1 $\beta$ synthesis (Il1b, Nlrp3, Klrb1a), we investigated whether JunD regulates active IL-1 $\beta$ secretion following Nlrp3-inflammasome activation in primary rat macrophages. Western Blot analysis of mature IL- $1 \beta$ in BMDMs primed with LPS and activated by ATP demonstrated a reduction in mature IL-1 $\beta$ between WKY and WKY.LCrgn2 BMDMs

Table 2 Microarray results of transcripts demonstrating greater than three-fold difference in expression between Jund siRNA and scrambled control siRNA transfected WKY BMDMs

\begin{tabular}{|c|c|c|c|c|c|}
\hline Gene symbol & Gene name & Chr. & Fold change & FDR (\%) & Gene Function \\
\hline \multicolumn{6}{|l|}{ Basal condition } \\
\hline Klrbla & killer cell lectin-like receptor subfamily B member 1B & 4 & 9.0 & $<0.01$ & IL-1 $\beta$ synthesis [19] \\
\hline$M t 2 A$ & metallothionein 2A & 19 & 4.6 & 0.63 & Oxidative stress [15] \\
\hline 1116 & interleukin 1 beta & 3 & 4.1 & 2.85 & IL-1 $\beta$ synthesis \\
\hline $\mathrm{CxCl9}$ & chemokine (C-X-C motif) ligand 9 & 14 & 3.5 & $<0.01$ & Inflammation \\
\hline $\operatorname{Len} 2$ & lipocalin 2 & 3 & 3.3 & $<0.01$ & Oxidative stress [16] \\
\hline Vcan & versican & 2 & 3.3 & $<0.01$ & Oxidative stress [17] \\
\hline Nlrp3 & NLR family, pyrin domain containing 3 & 10 & 3.2 & 3.01 & IL-1 $\beta$ synthesis [21] \\
\hline D3ZIY9_RAT & uncharacterised protein & 10 & 3.1 & 1.09 & - \\
\hline Dot11 & DOT1-like, histone H3 methyltransferase (S. cerevisiae) & 7 & -3.7 & 2.30 & Inflammation \\
\hline Hspb1 & heat shock protein 1 & 12 & -3.8 & $<0.01$ & Oxidative stress [18] \\
\hline Prkca & protein kinase C, alpha & 10 & -4.1 & 1.83 & Oxidative stress [19] \\
\hline \multicolumn{6}{|c|}{ LPS stimulated condition } \\
\hline Klrbla & killer cell lectin-like receptor subfamily B member 1B & 4 & 4.6 & $<0.01$ & IL-1 $\beta$ synthesis [20] \\
\hline Hspb1 & heat shock protein 1 & 12 & -4.0 & $<0.01$ & Oxidative stress [18] \\
\hline
\end{tabular}

Differentially expressed transcripts with greater than three-fold difference in expression (four technical replicates per strain) were identified at a $<5 \%$ FDR threshold following 40,000 permutations. Fold changes are of control siRNA versus Jund siRNA expression. A positive fold change indicates higher expression in BMDMs transfected with scrambled control siRNA i.e. with a higher level of Jund expression compared to Jund siRNA. Abbreviations: Chr.; chromosome, FDR: false discovery rate. 
Table 3 Gene ontology analysis for genes demonstrating differential expression over the eight hour LPS timecourse between WKY and WKY.LCrgn2 BMDMs

\begin{tabular}{|c|c|c|c|}
\hline Gene ontology term (BP_FAT or KEGG pathway) & Genes (n) & Fold Enrichment & Bonferroni corrected P-Value \\
\hline GO:0010033 response to organic substance & 84 & 2.23 & $9.00 \mathrm{E}-09$ \\
\hline GO:0009725 response to hormone stimulus & 53 & 2.57 & $1.59 \mathrm{E}-06$ \\
\hline GO:0009611 response to wounding & 46 & 2.68 & $6.71 \mathrm{E}-06$ \\
\hline GO:0009719 response to endogenous stimulus & 53 & 2.28 & $9.90 \mathrm{E}-05$ \\
\hline GO:0001775 cell activation & 31 & 3.18 & $1.08 \mathrm{E}-04$ \\
\hline GO:0043434 response to peptide hormone stimulus & 29 & 3.24 & $2.22 \mathrm{E}-04$ \\
\hline GO:0050865 regulation of cell activation & 25 & 3.63 & $2.44 \mathrm{E}-04$ \\
\hline GO:0002237 response to molecule of bacterial origin & 21 & 4.02 & $6.34 \mathrm{E}-04$ \\
\hline GO:0002694 regulation of leukocyte activation & 23 & 3.49 & 0.0018 \\
\hline GO:0032496 response to lipopolysaccharide & 19 & 3.95 & 0.0036 \\
\hline GO:0045767 regulation of anti-apoptosis & 11 & 7.37 & 0.0039 \\
\hline GO:0045321 leukocyte activation & 26 & 3.04 & 0.0039 \\
\hline GO:0001817 regulation of cytokine production & 22 & 3.42 & 0.0047 \\
\hline GO:0009617 response to bacterium & 26 & 3.00 & 0.0052 \\
\hline GO:0006954 inflammatory response & 26 & 2.98 & 0.0056 \\
\hline GO:0048545 response to steroid hormone stimulus & 31 & 2.65 & 0.0058 \\
\hline GO:0043067 regulation of programmed cell death & 54 & 1.96 & 0.0079 \\
\hline GO:0010941 regulation of cell death & 54 & 1.96 & 0.0085 \\
\hline GO:0031667 response to nutrient levels & 30 & 2.64 & 0.0095 \\
\hline GO:0042981 regulation of apoptosis & 53 & 1.95 & 0.012 \\
\hline GO:0009991 response to extracellular stimulus & 31 & 2.54 & 0.014 \\
\hline GO:0002684 positive regulation of immune system process & 26 & 2.80 & 0.018 \\
\hline GO:0051249 regulation of lymphocyte activation & 20 & 3.37 & 0.020 \\
\hline GO:0007568 aging & 20 & 3.35 & 0.022 \\
\hline GO:0006952 defense response & 36 & 2.28 & 0.024 \\
\hline GO:0045768 positive regulation of anti-apoptosis & 9 & 8.03 & 0.027 \\
\hline GO:0007565 female pregnancy & 16 & 3.94 & 0.033 \\
\hline GO:0014070 response to organic cyclic substance & 24 & 2.85 & 0.033 \\
\hline GO:0008283 cell proliferation & 27 & 2.62 & 0.036 \\
\hline GO:0046649 lymphocyte activation & 21 & 3.09 & 0.041 \\
\hline GO:0042592 homeostatic process & 53 & 1.86 & 0.044 \\
\hline
\end{tabular}

Enrichment for biological process functional annotation terms and KEGG canonical pathways used a Bonferroni corrected p-value threshold of $<0.05$. Abbreviations: BP_FAT, subset of Biological Process Gene Ontology (GO) terms generated by DAVID; $n$, number of involved genes.

Table 4 JunD binding events in WKY and WKY.LCrgn2 BMDMs

\begin{tabular}{lccc}
\hline \multicolumn{1}{c}{ Dataset } & $\begin{array}{c}\text { Total number of } \\
\text { peaks identified }\end{array}$ & $\begin{array}{c}\text { Number of peaks } \\
\text { linked to a gene }\end{array}$ & $\begin{array}{c}\text { Number of genes containing } \\
\text { at least one JunD peak }\end{array}$ \\
\hline WKY basal & 27124 & 12522 & 5339 \\
WKY LPS & 36687 & 18124 & 7612 \\
WKY.LCrgn2 basal & 16593 & 6408 & 2606 \\
WKY.LCrgn2 LPS & 8689 & 3361 & 1022 \\
\hline
\end{tabular}

Peaks were identified using BayesPeak v1.1.3 $[22,23]$ using a posterior probability threshold of 0.9 . Peaks were linked to a gene if they were located within 20 kilobases of the transcriptional start site or were located within the body of the gene. 
A

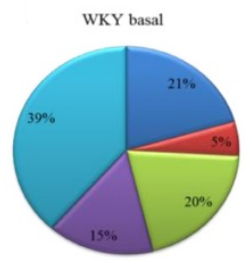

WKY.LCrgn2 basal

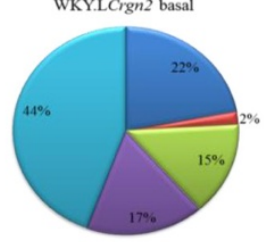

WKY LPS

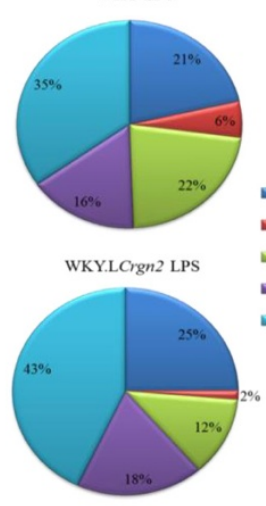

B

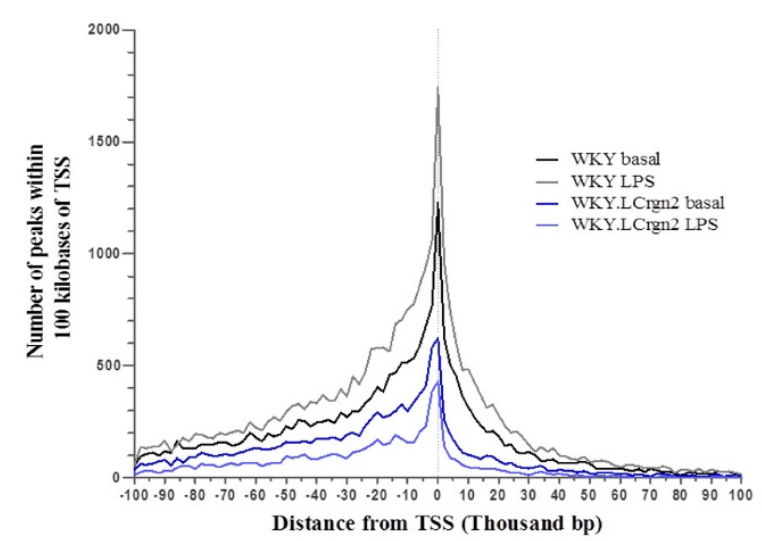

C

\begin{tabular}{|c|c|c|c|}
\hline 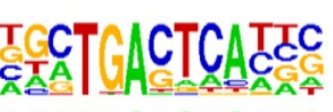 & $\begin{array}{l}\text { Transcription } \\
\text { factor }\end{array}$ & $\begin{array}{c}\% \text { peaks } \\
\text { containing } \\
\text { motif }\end{array}$ & P-value \\
\hline & AP-1 & 63.0 & $3.08 \mathrm{E}-87$ \\
\hline
\end{tabular}

D

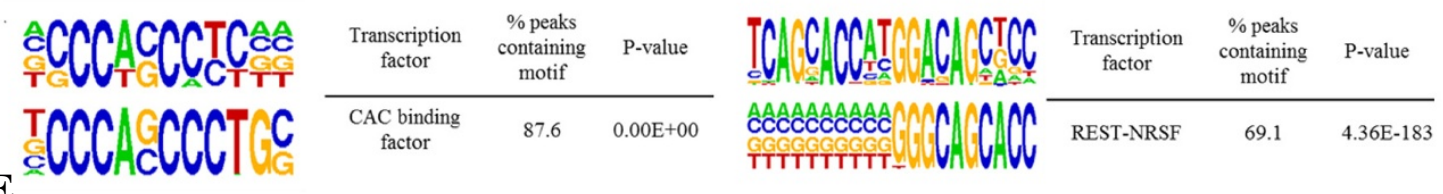

$\mathbf{E}$

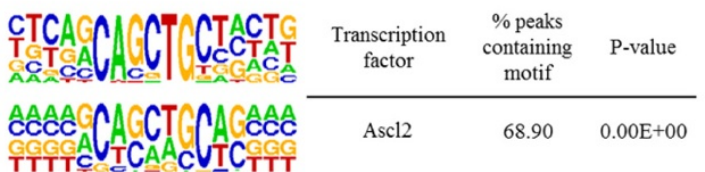

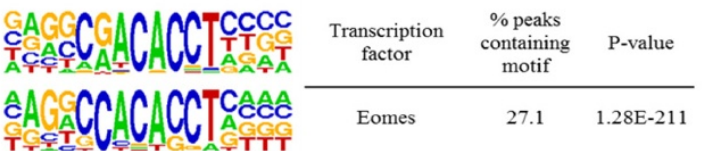

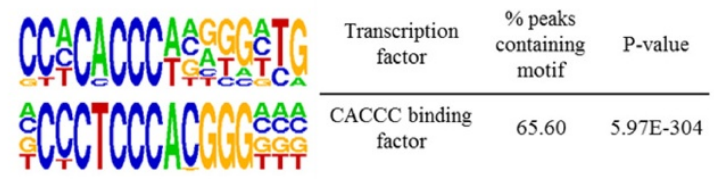

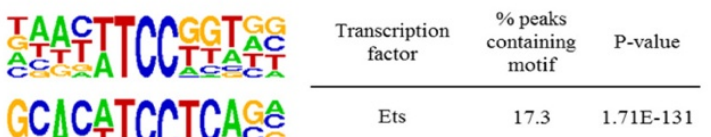

$\mathbf{F}$

Illb

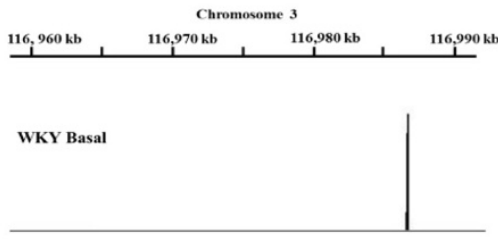

WKY.LCrgn2 Basal

HIH H

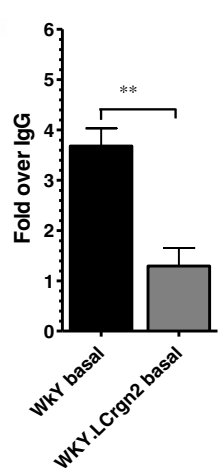

Strain and condition
Prkca

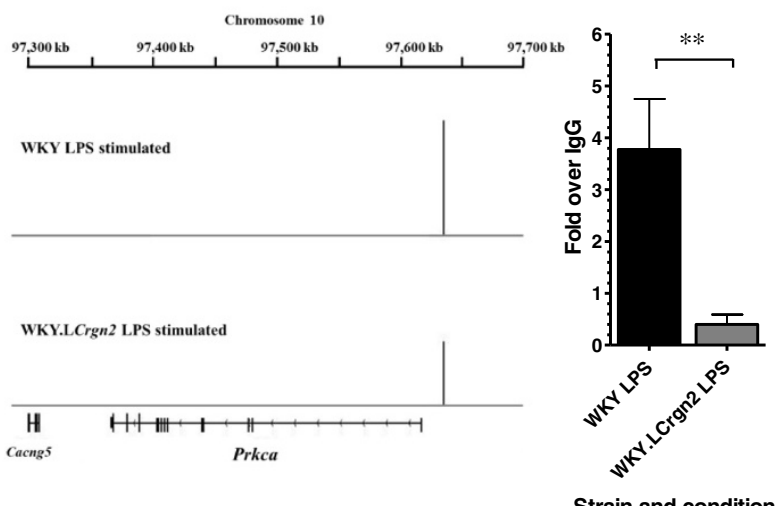

Strain and condition 
(See figure on previous page.)

Figure 4 Genetically determined differences in Jund expression alter the JunD cistrome and identifies primary JunD targets. (A) Distribution of JunD-peaks relative to transcriptional start sites (TSS) of Ensembl genes. Promoter region defined as 20 kilobases (kb) upstream of the TSS, upstream region between $10 \mathrm{~kb}$ and $50 \mathrm{~kb}$ upstream from TSS. (B) Occurrence of peaks within 100 kilobases of the TSS. (C) Twelve base pair AP-1 motif identified by de novo motif analysis present in 63\% peaks. De novo motif analysis using HOMER identified two de novo motifs in basal WKY BMDMs (D) and four motifs in LPS stimulated WKY BMDMs (E). The de novo motif identified is displayed on the bottom of each the pair of motifs, the matched consensus motif for a transcription factor on the top. (F) //1b and Prkca confirmed as primary JunD targets by qPCR validation. The aligned reads comprising peak passing the posterior probability threshold of 0.9 for each JunD-bound gene in the WKY strain in the LPS stimulated state for 116 and the basal state for Prkca are shown in genome browser views along with the peak in the WKY.LCrgn2 strain. Samples from WKY and WKY.LCrgn2 strains were amplified using three biological replicates with three technical replicates per sample. Results expressed as mean fold change over lgG. ${ }^{*} \mathrm{P}<0.05$; ${ }^{*} \mathrm{P}<0.01$; using a one-tailed unpaired t-test to detect statistically significant differences between the strain and condition pairs. Error bars represent standard error of the mean.

(Figure 6A). Importantly, there was a marked increase in mature IL-1 $\beta$ production in LEW.WCrgn2 BMDMs compared to LEW confirming that the Jund congenic interval was able to alter mature IL- $1 \beta$ production (Figure $6 \mathrm{~A}$ ). These results were confirmed by ELISA for IL-1 $\beta$ in LPS primed and ATP stimulated BMDMs which showed significant differences between the production of IL-1 $\beta$ by WKY BMDMs compared to WKY.LCrgn2 and LEW BMDMs (Figure 6B). The production of IL-1 $\beta$ in nephritic glomeruli from WKY, WKY.LCrgn2, LEW and LEW. WCrgn 2 rats was examined (Figure 6C). This showed a significant reduction in IL- $1 \beta$ production in all the other strains compared to WKY demonstrating a role for the Crgn 2 congenic interval in IL-1 $\beta$ production.

\section{Discussion}

The aim of the present study was to investigate genes regulated by JunD mediating macrophage oxidative burst and pro-inflammatory cytokine production leading to enhanced cell activation in the WKY rat. We used combined microarray and JunD/AP1 ChIP-Seq analyses in primary BMDMs from WKY (NTN-susceptible, high JunD levels, enhanced macrophage oxidative burst) and congenic WKY.LCrgn2 (reduced NTN, reduced JunD levels, reduced macrophage oxidative burst) rats. Microarray analysis was performed in two experimental settings: following Jund siRNA knockdown using lipid-based transfection in WKY BMDMs and between WKY and WKY. LCrgn2 primary macrophages. ChIP-Seq analysis was also performed between WKY and WKY.LCrgn2 BMDMs in order to identify primary JunD targets. Microarray and ChIP-Seq experiments were also performed in macrophages activated with LPS to assess the role of JunD in macrophages activated through LPS/TLR4.

JunD reduces tumour angiogenesis by limiting Rasmediated production of reactive oxygen species (ROS) implicated in the pathophysiology of various diseases, including cancer, regulates genes involved in antioxidant defence and enhances the transcription of VEGF-A, a potent proangiogenic factor $[7,9]$. In addition JunD deficient mice display persistent hyperinsulinaemia resulting from enhanced pancreatic islet vascularization owing to chronic oxidative stress [9]. In crescentic glomerulonephritis JunD deficiency may cause increased oxidative stress in the glomerular podocytes, leading to altered VEGFA expression and subsequent glomerular injury [10]. In the rat NTN model of Crgn, reduced JunD expression in the congenic WKY.LCrgn2 strain is associated with $11 \%$ reduction in glomerular crescent formation [12,13]. We have carried out a combined ChIP-Seq and transcriptome approach in macrophages, the main effector cells of Crgn, in order to identify JunD targets that may explain its modulatory role. Alongside a general effect by JunD to alter the immune response to LPS in the overall gene sets, the functions of strongly dysregulated ( $>3$ fold) expression changes suggested that primary gene targets of JunD are key effectors in mediating protection from oxidative stress and IL- $1 \beta$ synthesis. Interestingly, this approach identified genes regulating oxidative stress that were previously identified to be under the regulation of JunD in fibroblasts (i.e. cysteine dioxygenase [7]) suggesting that JunD may have common targets in the oxidative stress pathway in different cell types. JunD regulates IL- $1 \beta$ secretion in rat BMDMs and pro-inflammatory cytokine secretion in human monocyte-derived macrophages [12] suggesting that its role of the regulation of the M1 macrophage activation is conserved across the species. Taken together these observations suggest that JunD regulates oxidative stress various diseases with both common and cell specific targets.

Current understanding of JunD function on a genome wide scale has been limited by studies performed on a candidate gene or promoter basis [24-26]. The Encyclopaedia of DNA elements (ENCODE) project has carried out ChIP-Seq for JunD in human transformed cell lines though not in a macrophage or monocyte cell lines [27-29]. JunD has been categorised as a middle-level transcription factor and such factors regulate information-flow bottlenecks and may be the best therapeutic targets for strongly affecting the flow of information through regulatory circuits [29]. The cistrome of JunD was also found to be highly context and cell type specific $[29,30]$. We used primary macrophages from two inbred rat 


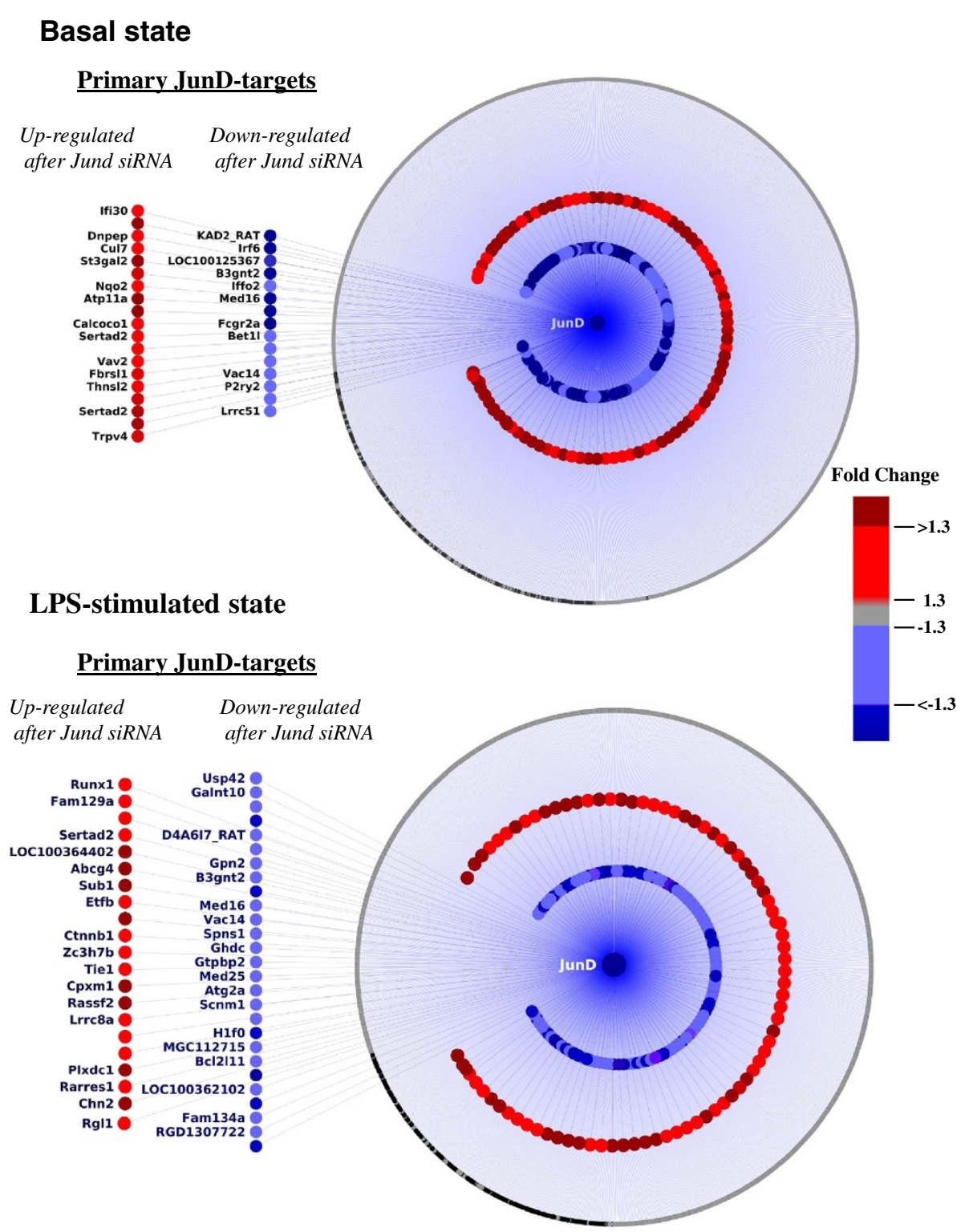

Figure 5 Integrative analysis identifies primary JunD targets in basal and LPS stimulated BMDMs. Jund gene expression patterns in WKY and WKY.LCrgn2 BMDMs over the LPS time course were used for Spearman correlation analysis with the rest of the transcripts on the microarrays ( $P<0.001$ cut off). The correlated genes were used for building the JunD target gene networks and selected based on i. significant differential expression following Jund siRNA knockdown (both basal and after LPS stimulation FDR $<5 \%$ ), ii. presence of the JunD ChIP-Seq peak in WKY BMDMs (both basal and after LPS stimulation). The outer ring represents all the transcripts correlating with Jund expression levels $(P<0.001)$ in WKY and WKY.LCrgn2 BMDMs (1445 transcripts, 75\%, indicated in grey circles) over the LPS time course. Transcripts associated with a JunD ChIPSeq peak (within 20kb of TSS or within the gene body) are shown as black circles (232 transcripts, 12\%). The transcripts correlating with Jund expression levels and down-regulated following Jund siRNA (basal, upper panel) are shown in blue (125 transcripts, 6.5\%); and those up-regulated (basal, upper panel) are shown as red circles (116 transcripts, 6\%). Primary JunD targets in basal BMDMs are given with the gene names and show a JunD ChIP-Seq peak (24 transcripts, basal state, upper panel). This analysis was repeated for the LPS-stimulated macrophages taking into account JunD siRNA and ChIP-Seq datasets in LPS stimulated macrophages and identified 36 primary Jund targets (lower panel).

strains expressing different amounts of JunD in a comparative ChIP-Seq analysis to identify the genomic regions uniquely bound by JunD. The overall landscape of JunD binding in the BMDMs from WKY and WKY. LCrgn2 BMDMs was comparable to that of other transcription factors studied in primary macrophages stimulated by LPS [31,32]. We found that after LPS stimulation in WKY BMDMs, there was enrichment for genes involved in multiple immune processes linked with responses to multiple different stimuli. In combination with the gene expression data, these findings suggest that genetically determined up-regulation of JunD 

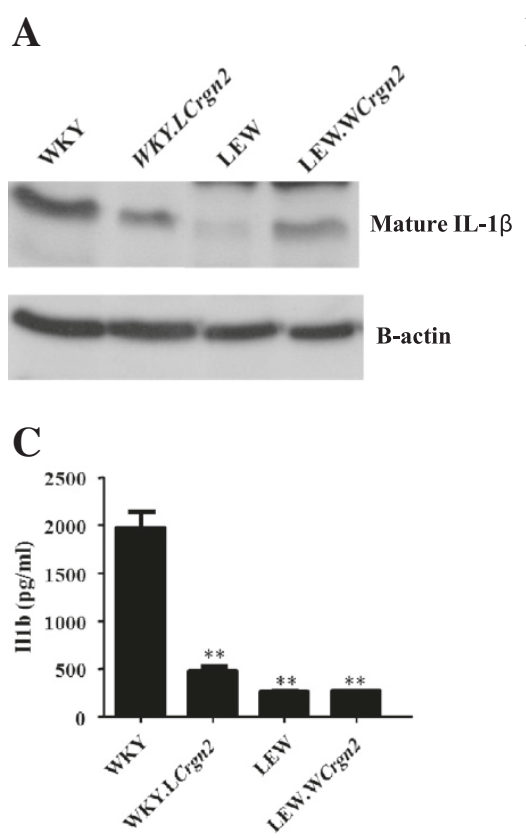

B

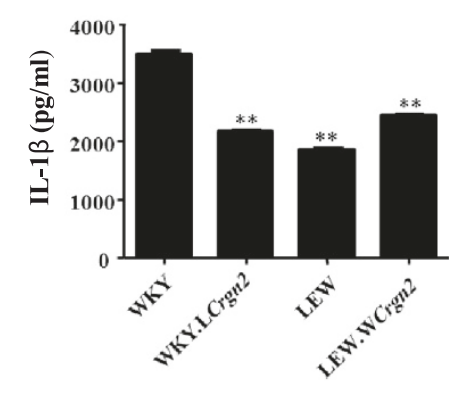

Figure 6 JunD expression levels control the production of mature IL-1 $\beta$ in BMDMs and nephritic glomeruli. (A) Western blot of mature IL-1 $\beta$ expression in WKY, WKY.LCrgn2, LEW and LEW.WCrgn2 BMDMs primed with LPS and stimulated with ATP. The result of 3 independent experiments is demonstrated alongside $\beta$-actin loading control. ELISAs for IL-1 $\beta$ in LPS primed and ATP activated BMDMs (B) and in nephritic glomeruli $(\mathbf{C}) .{ }^{*} \mathrm{P}<0.01$ statistically significantly different to WKY using a one way-ANOVA with Bonferonni's post-tests.

expression resulted in enhanced macrophage activation in the WKY strain.

De novo motif analysis identified additional transcription factor motifs that were unique to the WKY strain suggesting that the increased levels of JunD expression facilitated new partnerships with other transcription factors that did not occur in WKY.LCrgn2 BMDMs. Key findings included the CACCC-binding domain which binds Krüppel-like family (KLF) transcription factors and are regulators of signalling following activation of macrophages [33-35] and the Ets-1 motif, a factor controlling the expression of cytokine and chemokine genes in a wide variety of cells [36] in LPS stimulated WKY BMDMS. In basal WKY BMDMS a RESTNRSF motif was identified consistent with the functional findings of neuron development and differentiation in unique core JunD-bound genes in WKY BMDMS and potential roles for JunD in excitoxic neuronal cell death and ischaemic injury $[37,38]$.

The main goal of the study was to identify primary JunD target genes responsible for the macrophage activation seen in Crgn-susceptible WKY rat. The siRNA knockdown experiments identified that genes with the greatest changes in expression were associated with IL-1 $\beta$ synthesis. The transcriptional control of $I l 1 b$ expression by JunD was further confirmed by investigating IL-1 $\beta$ secretion upon inflammasome activation in the WKY and reciprocal Jund congenic strain BMDMs and nephritic glomeruli (WKY.LCrgn2 and LEW.WCrgn2).
Our integrative analysis identified primary JunD targets through which JunD could primarily regulate macrophage activation. In the basal state multiple targets have links with oxidative stress including Trpv4 [39], Vav2 [40-42], Ifi30 [43], Nqo2 [44] and P2ry2 [45]. This was also seen after LPS stimulation with transcripts such as Ctnnb1 [46] and Bcl2l11 [47] highlighting the key role of JunD in the regulation of oxidative stress in WKY BMDMs. Moreover, the transcription factor Run $x 1$ (a target in the LPS stimulation group) has been identified as a primary JunD target suggesting that novel transcription factor interactions in macrophages may underlie some JunD-mediated macrophage activation.

\section{Conclusions}

Taken together our data show that genetically determined differences in physiological levels of JunD affect its genome-wide binding patterns in basal and LPSstimulated primary macrophages. These results identified transcriptional programs underlying JunD-mediated oxidative stress and IL-1 $\beta$ synthesis in primary macrophages which may play a role in susceptibility to Crgn.

\section{Methods}

\section{Animals}

WKY (WKY/NCrl) and LEW (LEW/Crl) rats were purchased from Charles River (Margate, UK). Single congenic rats were generated by introgressing the $\mathrm{Crgn} 2$ 
QTL from chromosome 16 from a LEW donor onto a WKY recipient background and vice versa as previously described [12]. All procedures were performed in accordance with the United Kingdom Animals (Scientific Procedures) Act.

\section{BMDM culture}

BMDMs were prepared from the femurs of parental and congenic strains using previously described methods [12]. Femurs from adult (8-10 weeks) rats were isolated and flushed with Hanks buffer (Life Technologies). Total bone marrow derived cells were plated and cultured for 5 days in Dulbecco's modified Eagle's medium (Life Technologies) containing $25 \mathrm{mM}$ Hepes (Sigma), 25\% L929 conditioned medium, 25\% decomplemented fetal bovine serum (Biosera), penicillin $(100 \mathrm{U} / \mathrm{ml}$, Invitrogen), streptomycin $(100 \mu \mathrm{g} / \mathrm{ml}$, Invitrogen $)$ and ${ }_{\mathrm{L}}$-glutamine (2 mM Invitrogen). The cells were characterised as macrophages by ED-1 staining. Basal macrophages were left unstimulated whilst stimulated cells were stimulated with $100 \mathrm{ng} / \mathrm{ml}$ lipopolysaccharide (Sigma). Following stimulation, cells for gene expression analysis were homogenized in TRIzol (Invitrogen) and stored at $-80^{\circ} \mathrm{C}$.

\section{siRNA inhibition of Jund expression}

siRNA knockdown was carried out as previously described [12]. Briefly, on day 5 of culture, WKY BMDMs were replated in six-well plates $\left(1 \times 10^{6}\right.$ cells per well) in DMEM (Invitrogen) overnight and transfected for 48 hours with siGENOME SMARTpool for Jund (100 nM, Dharmacon) or siGENOME non-targeting siRNA pool as the scrambled control siRNA using Dharmafect 1 (1:50, Dharmacon) as a transfection reagent in OPTIMEM medium (Invitrogen). The siRNA sequences used in the siGENOME SMARTpool for Jund are listed in Additional file 2: Table S7. siRNA knockdown was confirmed with quantitative PCR (detailed below) and Western blotting (Figure 1).

\section{RNA extraction and microarray preparation}

Total RNA was extracted using the TRIzol method and purified using RNeasy Plus spin columns (Qiagen). 100ng of RNA was amplified, labelled and hybridised to Rat Gene 1.0 ST arrays (Affymetrix, Santa Clara, CA, USA) using the Ambion WT Expression Kit (Life Technologies) as per manufacturer's instructions. For timecourse expression analysis, four BMDM preparations from four biological replicates were used for each timepoint and condition. For siRNA expression analysis, four BMDM preparations from at least two biological replicates were used for each timepoint and condition. The microarray data is available in MIAME-compliant (minimum information about a microarray experiment) format at the Array Express database (http://www.ebi.ac. uk/arrayexpress) under accession code E-MEXP-3469.

\section{Microarray data analysis}

CEL intensity files were produced using GeneChip Operating Software version 1.4 (Affymetrix) and quality tested using the Affymetrix Expression Console v1.1.2. All 32 files in the timecourse data set and 16 files in the siRNA dataset were suitable for further analysis. Probelevel data was normalised using robust multichip average (RMA) [48,49]. A custom definition file was created using up-to-date probe information [50] and filtered to exclude probes containing the 2,520,602 single nucleotide polymorphisms present between the WKY and LEW genomes (Santosh Atanur, MRC Clinical sciences centre, personal communication). The moderated $\mathrm{T}$ test with 40,000 permutations implemented in Statistical Analysis of Microarrays (SAM) version 3.0 was used to identify differentially expressed genes at an FDR threshold of 5\% and timecourse analysis was performed using EDGE with 40,000 permutations and a 5\% FDR threshold [51]. Hierarchical clustering analysis was performed using MultiExperiment Viewer $(\mathrm{MeV})$ v4.8 [52,53] with the Euclidean distance measure. Gene ontology analysis was carried out using the functional annotation tools within DAVID, the Database for Annotation, Visualisation and Integrated Discovery v6.7 [54,55].

\section{Quantitative PCR}

All qPCRs were performed with an ABI 7900 Sequence Detection System (Applied Biosystems, Warrington, UK). A two-step protocol was used as previously described [56] beginning with cDNA synthesis with iScript select (Bio-Rad) followed by PCR using SYBR Green Jumpstart Taq Ready Mix (Sigma). A total of 10 ng of cDNA per sample was used. All samples were amplified using a set of 4 biological replicates with three technical replicates used per sample in the PCR. Sequence detection software (SDS) version (Applied Biosystems) was used to obtain the $\mathrm{Ct}$ values. Results were analysed using the comparative $\mathrm{Ct}$ method and each sample was normalised to the reference gene Hprt, to account for any cDNA loading differences. The primer sequences used for the qRT-PCR validation of microarray data are listed in Additional file 2: Table S8.

\section{Chromatin immunoprecipitation (ChIP)}

BMDMs were left in the basal condition or stimulated for 2 hours with 100ng/ml LPS. Cells were fixed for 10 minutes with $1 \%$ formaldehyde and ChIP performed using ChIP-IT Express as per manufacturer's instructions with some modifications. Sonication was carried out using Covaris S2 (Woburn, Massachusetts, USA) in a volume of $300 \mu \mathrm{l}$ sonication buffer with the following 
settings; 20\% duty cycle, intensity 8, 200 cycles/burst, cycle length $30 \mathrm{~s}$ for 28 to 30 cycles dependant on cell count. The ChIP lysate was immunoprecipitated using 2 $\mu \mathrm{g}$ of JunD antibody (Santa Cruz sc74-X) or negative IgG control (sc-2026) overnight. Cross links in the immunoprecipitated chromatin and control input chromatin were reversed by heating the samples at $65^{\circ} \mathrm{C}$ for 5 hours followed by proteinase $\mathrm{K}$ digestion for 1 hour. Samples were purified using Qiagen MinElute columns as per manufacturer's instructions prior to downstream analysis.

\section{High throughput sequencing}

Single read library preparation and high throughput single read sequencing for 36 cycles was carried out on an Illumina Genome Analyser IIx according to the manufacturer's protocols (Illumina) with some modifications. Each immunoprecipitated sample for library preparation was the product of five separate technical replicates of immunoprecipitation pooled together and purified using Qiagen MinElute columns and quantified using the hsDNA Qubit assay (Invitrogen, Life Technologies, California, USA). A 1:20 dilution of Illumina adaptors was used at the adaptor ligation step to avoid adaptor dimer formation and a 1:2 primer dilution used to prevent dimerization during the PCR amplification stage. The samples were quantified using the hsDNA Qubit assay (Invitrogen, Life Technologies, California, USA) and the size range analysed using a HS DNA chip on a 2100 Bioanalyser (Agilent Technologies, West Lothian, UK) prior to submission for $\mathrm{qPCR}$ analysis and cluster generation and sequencing by the CSC/IC Genome Core facility.

\section{qPCR of ChIP enriched DNA}

Immunoprecipitated DNA fragments were analysed by real-time PCR. Primers used are listed in Additional file 2: Table S9. All samples were amplified using a set of 3 biological replicates with three technical replicates used per sample. After an initial denaturation step of $94^{\circ} \mathrm{C}$ for 2 mins, the samples were cycled 40 times at $94^{\circ} \mathrm{C}$ for $15 \mathrm{~s}$, $60^{\circ} \mathrm{C}$ for $1 \mathrm{~min}$ and $72^{\circ} \mathrm{C}$ for $1 \mathrm{~min}$ with data collection performed during the $72^{\circ} \mathrm{C}$ elongation step. Sequence detection software (SDS) version 2.3 (Applied Biosystems) was used to obtain the $\mathrm{Ct}$ values and each sample was analysed with reference to $\mathrm{Ct}$ values for matched control 'Input' non-immunoprecipitated chromatin. A standard curve of 1:5 dilutions of Input DNA was used to calculate the \% Input level of the transcription factor binding at the investigated locus. The standard curve was constructed from the Input DNA sample for the appropriate strain and condition and analysed within the SDS software v2.3. For each test gene the \% Input levels were then determined using \%total $=2^{\Delta \mathrm{Ct}} \times(\%$ of input sample used) where $\Delta=\mathrm{Ct}$ (Input) - Ct (sample). Fold change over IgG was expressed using $2^{-\Delta \mathrm{Ct}}$ where $\Delta \mathrm{Ct}=\Delta \mathrm{Ct}_{\text {Jund }}-\Delta \mathrm{Ct}_{\mathrm{IgG}}$.

\section{ChIP-Seq data analysis}

Sequencing of the ChIP-Seq libraries was carried out on the high throughput Illumina Genome Analyzer II. Initial data processing was performed using Illumina Real Time Analysis (RTA) v1.6.32 software (equivalent to Illumina Consensus Assessment of Sequence and Variation, CASAVA 1.6) with default filter and quality settings. Quality filtered reads were then realigned to the reference rat genome (RGSC3.4) using the Burrows Wheeler Alignment tool v0.5.9 (BWA) [57]. Read ends were trimmed if Phred-scaled base quality scores dropped below 20. Reads that uniquely mapped to the reference genome were used to detect areas of enrichment with BayesPeak v1.1.3 [22,23] using a posterior probability threshold of 0.9. A stringent posterior probability threshold of 0.9 was used to filter all bins passing the threshold to form the final contiguous peak regions to produce a more accurate reflection of true peak calls. An over fitting diagnostic was performed using $\lambda_{1}<0.7$ and score $<-2.25$ to filter out regions which showed no enrichment but had a high enough background for the algorithm to call peaks in. Peak regions were annotated using the gene intervals annotator (GIN) implemented in CARPET using a gene priority approach to give the associated transcript I.D., the associated gene feature and the distance of the peak to the nearest transcriptional start site [58]. HOMER was used to predict motif occurrence within peaks $[32,59]$ with default settings for a maximum motif length of 12 base pairs. The outputs of the peak calling algorithms were visualised in the Integrative Genomics Viewer [60] using custom WIG files generated from the output data generated by BayesPeak. The latter were generated by extending each mapped read by 200 bp and then by using 10 bp bins the overlapping tag count was generated.

\section{Integrated data analysis and identification of primary JunD targets}

JunD gene expression patterns in WKY and WKY. LCrgn2 BMDMs over the LPS time course were used for Spearman correlation analysis with the rest of the transcripts on the microarrays ( $\mathrm{P}<0.001$ cut off). In order to integrate the three different datasets, the list of significantly correlated set of transcripts was filtered and annotated according to two criteria: significant differential expression following Jund siRNA knockdown (both basal and after LPS stimulation) and the presence of the JunD ChIP-Seq peaks in WKY BMDMs (both basal and after LPS stimulation). Two separate networks (basal and LPS) were built with Cytoscape version 2.8.3 showing primary JunD targets. 


\section{Isolation and culture of rat nephritic glomeruli and detection of IL-1 $\beta$ by Western Blot and ELISA}

Glomeruli were isolated as previously described [13]. After 48 hours of incubation at $37^{\circ} \mathrm{C}$, nephritic glomeruli supernatants were collected and stored at $-20^{\circ} \mathrm{C}$ for IL$1 \beta$ sandwich ELISA analysis. For IL-1 $\beta$ detection in BMDMs by Western Blotting, cells were primed with LPS $(1 \mu \mathrm{g} / \mathrm{ml}$, 3hours $)$ and stimulated with ATP $(5 \mathrm{mM})$ for 30 minutes. BMDMs were then scraped and both cells and supernatant were collected, filtered using Amicon ultra centrifugal filters for protein purification and concentration (10 kDa cut-off, Millipore, UK) and the concentrated samples were diluted with $5 \times$ sample buffer containing $200 \mathrm{mM}$ Tris- $\mathrm{HCl}$, 6\% SDS, 2mM EDTA, $4 \%$ 2- Mercaptoethanol, $10 \%$ glycerol and boiled for 10 minutes. The samples were then resolved by SDS polyacrylamide gel electrophoresis (PAGE) and transferred to an Immobilon-P Transfer Membrane (Millipore). Rabbit polyclonal anti IL-1 $\beta$ (New England BioLabs, UK) was used to detect the mature IL-1 $\beta$. To assess secreted IL-1 $\beta$ in BMDMs and nephritic glomeruli, cell supernatants were subjected to sandwich ELISA and secreted IL-1 $\beta$ amounts were determined using a standard curve with rat recombinant IL-1 $\beta$ according to manufacturer's instructions $(\mathrm{R}$ and $\mathrm{D}$ Systems).

For the confirmation of siRNA knockdown of Jund, WKY BMDMs were plated into six-well plates at a density of $1 \times 10^{6}$ cells per well and treated with Jund specific siRNA or a scrambled oligonucleotide for 48 hours before total protein was extracted for Western Blot analysis using the above technique. For JunD detection a specific rabbit polyclonal anti-Jund antibody from Santa Cruz Biotechnology (USA) was used. This blot is representative of 4 different experiments performed with 4 biological replicates.

\section{Additional files}

Additional file 1: Figure S1. Genome-wide expression analysis in basal and LPS stimulated BMDMs. Genome wide expression analysis by microarrays was performed in BMDMs transfected with rat Jund or scrambled control siRNA for the unstimulated condition (A) or following eight hours of LPS stimulation (B) in WKY BMDMs and over an eight hour time course of LPS stimulation in WKY and WKY.LCrgn2 BMDMs (C). Heat maps of hierarchically clustered significantly differentially expressed genes ( $<5 \%$ FDR threshold) are displayed. All experiments were performed in 4 biological replicates for each strain or siRNA transfected. Figure S2. Validation of microarray data between WKY and WKY.LCrgn2 BMDMs over an eight hour LPS stimulation timecourse. Validation of microarray data by qRT-PCR. Samples were amplified using a set of four biological replicates with three technical replicates per sample. Relative gene expression was measured by GRT-PCR and normalised with Hprt for WKY and WKY.LCrgn2 BMDMs. ${ }^{*} P<0.05 ;{ }^{*} \mathrm{P}<0.01$; ${ }^{* *} \mathrm{P}<0.001$ statistically significantly different to WKY using a two way ANOVA to compare the overall timecourse with Bonferonni's post-tests to compare individual time points. Figure S3. ChIP-Seq peak validations by ChIP-qPCR. ChIPSeq peaks identified at a posterior probability threshold of 0.9 for basal
WKY BMDMs were validated by GPCR (A) and for LPS stimulated WKY BMDMs (B) and WKY.LCrgn2 BMDMs peaks (C). Samples were amplified using a set of biological triplicates with three technical replicates per sample. Results expressed as mean fold change over lgG. ${ }^{* *} \mathrm{P}<0.01$, ${ }^{*} \mathrm{P}<0.05$, ns; non-significant using a paired t-test (one-tailed) to compare whether \% input for the JunD ChIP qPCR was significantly different to \% input for IgG. Figure S4. $/ / 16$ and Prkca confirmed as primary JunD targets by qPCR validation. The aligned reads comprising peak passing the posterior probability threshold of 0.9 for each JunD-bound gene in the WKY strain in the basal state for 116 (A) and the LPS stimulated state for Prkca (B) are shown in genome browser views along with the peak in the WKY.LCrgn2 strain. Samples from WKY and WKY.LCrgn2 strains were amplified using three biological replicates with three technical replicates per sample. Results expressed as mean fold change over lgG. ${ }^{*} \mathrm{P}<0.05$; ${ }^{*} \mathrm{P}<0.01$; using a one-tailed unpaired t-test to detect statistically significant differences between the strain and condition pairs. Error bars represent standard error of the mean. Figure S5. Integrative analysis identifies the transcription factor $B C / 2 / 11$ as a primary JunD target. Jund microarray-determined expression patterns in WKY and WKY.LCrgn2 BMDMs over an eight hour LPS timecourse using four biological replicates per strain were used for Spearman correlation analysis (A) with the rest of the transcripts on the microarrays. The expression of $B C / 2111$ (B) was significantly correlated to the Jund expression pattern (Spearman correlation 0.9 , corrected $p$-value $\left.=8.6 \times 10^{-5}\right)$. Significant differential expression of the gene was seen following siRNA knockdown of Jund (C). Fold changes are of control siRNA versus Jund siRNA expression. The positive fold change indicates higher expression in BMDMs transfected with scrambled control siRNA i.e. with a higher level of Jund expression compared to Jund siRNA. Abbreviations: Chr.; chromosome, FDR: false discovery rate. Three JunD binding events were identified at a posterior probability threshold of 0.9 in LPS stimulated WKY BMDMs (D) located in the gene promoter and second intron.

Additional file 2: Table S1. Validation of differentially expressed genes identified by siRNA microarray data analysis with quantitative PCR. Table S2. Sequencing and mapping statistics for ChIP-Seq in WKY and WKY.

LCrgn2 BMDMs. Table S3. Gene ontology analysis of JunD-bound genes in basal WKY BMDMs. Table S4. Gene ontology analysis of JunD-bound genes in basal WKY.LCrgn2 BMDMs. Table S5. Gene ontology analysis of JunD-bound genes in LPS stimulated WKY.LCrgn2 BMDMs. Table S6. Gene ontology analysis of JunD-bound genes in LPS stimulated WKY BMDMs. Table S7. Sequences of the four individual siRNAs that comprise siGENOME SMARTpool M-092127-00-0010 (Dharmacon). Table S8. Primer sequences used for qRT-PCR validation of microarray data. Table S9. Primer sequences used for qPCR validation of ChIP-Seq data.

\section{Competing interests}

The authors declare that they have no competing interests.

\section{Authors' contributions}

$\mathrm{RPH}, \mathrm{THC}$, TJA and JB conceived and designed the study. RPH, ZD and JB carried out the laboratory experiments and LG led the microarray and high throughput sequencing laboratory analyses. PKS and RPH carried out the bioinformatic analysis with contributions from SSA and EP. JB wrote the manuscript with RPH and contributions from TJA, THC and FMG. All authors read and approved of the final manuscript.

\section{Acknowledgements}

We thank Ailsa Chiu and CSC/IC Genome Core facility for their excellent technical assistance. This work was primarily supported by a Wellcome Trust Clinical PhD Fellowship (087182/Z/08/Z to RPH), a Junior Fellowship from Imperial College (to JB), by intramural funding from the MRC Clinical Sciences Centre (to TJA) and by the Wellcome Trust project grant (WT092523MA to JB).

\section{Author details}

${ }^{1}$ MRC Clinical Sciences Centre, Imperial College London, Hammersmith hospital, Du Cane Road W12 0NN, London, UK. ${ }^{2}$ Stress and Cancer Laboratory, Institut Curie, 26 Rue d'Ulm, Paris, France. ${ }^{3}$ Centre of Complement and Inflammation Research, Imperial College London, Du Cane Road W12 0NN, London, UK. 
Received: 13 October 2012 Accepted: 1 February 2013

Published: 11 February 2013

\section{References}

1. Mosser DM, Edwards JP: Exploring the full spectrum of macrophage activation. Nat Rev Immunol 2008, 8(12):958-969.

2. Dale DC, Boxer L, Liles WC: The phagocytes: neutrophils and monocytes. Blood 2008, 112(4):935-945.

3. Shaulian E: AP-1 - The Jun proteins: oncogenes or tumor suppressors in disguise? Cell Signal 2010, 22(6):894-899.

4. Shaulian E, Karin M: AP-1 in cell proliferation and survival. Oncogene 2001, 20:2390-2400

5. Wagner EF, Eferl R: Fos/AP-1 proteins in bone and the immune system. Immunol Rev 2005, 208(1):126-140.

6. Olefsky JM, Glass CK: Macrophages, inflammation, and insulin resistance. Annu Rev Physiol 2010, 72:219-246.

7. Gerald D, Berra E, Frapart YM, Chan DA, Giaccia AJ, Mansuy D, Pouysségur J, Yaniv M, Mechta-Grigoriou F: JunD Reduces Tumor Angiogenesis by Protecting Cells from Oxidative Stress. Cell 2004, 118(6):781-794.

8. Toullec A, Gerald D, Despouy G, Bourachot B, Cardon M, Lefort S, Richardson M, Rigaill G, Parrini MC, Lucchesi C, et al: Oxidative stress promotes myofibroblast differentiation and tumour spreading. EMBO MOl Med 2010, 2(6):211-230.

9. Laurent G, Solari F, Mateescu B, Karaca M, Castel J, Bourachot B, Magnan C, Billaud M, Mechta-Grigoriou F: Oxidative Stress Contributes to Aging by Enhancing Pancreatic Angiogenesis and Insulin Signaling. Cell Metab 2008, 7(2):113-124

10. Cook HT, Tarzi R, D'Souza Z, Laurent G, Lin W-C, Aitman TJ, MechtaGrigoriou F, Behmoaras J: AP-1 Transcription Factor JunD Confers Protection from Accelerated Nephrotoxic Nephritis and Control Podocyte-Specific Vegfa Expression. Am J Pathol 2011, 179(1):134-140.

11. Tam F, Smith J, Morel D, Karkar A, Thompson E, Cook H, Pusey C: Development of scarring and renal failure in a rat model of crescentic glomerulonephritis. Nephrol Dial Transplant 1999, 14(7):1658-1666.

12. Behmoaras J, Bhangal G, Smith J, McDonald K, Mutch B, Lai PC, Domin J, Game L, Salama A, Foxwell BM, et al: Jund is a determinant of macrophage activation and is associated with glomerulonephritis susceptibility. Nat Genet 2008, 40(5):553-559.

13. Behmoaras J, Smith J, D'Souza Z, Bhangal G, Chawanasuntoropoj R, Tam FW, Pusey CD, Aitman TJ, Cook HT: Genetic loci modulate macrophage activity and glomerular damage in experimental glomerulonephritis. J Am Soc Nephrol 2010, 21(7):1136-1144.

14. Smolinska MJ, Page TH, Urbaniak AM, Mutch BE, Horwood NJ: Hck Tyrosine Kinase Regulates TLR4-Induced TNF and IL-6 Production via AP-1. $\mathrm{J}$ Immunol 2011, 187(11):6043-6051.

15. Reinecke F, Levanets O, Olivier $Y$, Louw R, Semete B, Grobler A, Hidalgo J, Smeitink J, Olckers A, FH Vdw: Metallothionein isoform 2A expression is inducible and protects against ROS-mediated cell death in rotenonetreated HeLa cells. Biochem J 2006, 395(2):405-415.

16. Roudkenar MH, Halabian R, Ghasemipour Z, Roushandeh AM, Rouhbakhsh M, Nekogoftar M, Kuwahara Y, Fukumoto M, Shokrgozar MA: Neutrophil Gelatinase-associated Lipocalin Acts as a Protective Factor against $\mathrm{H} 2 \mathrm{O} 2$ Toxicity. Arch Med Res 2008, 39(6):560-566.

17. Wu Y, Wu J, Lee DY, Yee A, Cao L, Zhang Y, Kiani C, Yang BB: Versican protects cells from oxidative stress-induced apoptosis. Matrix Biol 2005, 24(1):3-13.

18. Mymrikov EV, Seit-Nebi AS, Gusev NB: Large Potentials of Small Heat Shock Proteins. Physiol Rev 2011, 91(4):1123-1159.

19. Fontayne A, Dang PM-C, Gougerot-Pocidalo M-A, El Benna J: Phosphorylation of p47phox Sites by PKC $a, \beta I I, \delta$, and $\zeta$ : Effect on Binding to p22phox and on NADPH Oxidase Activation. Biochemistry 2002, 41(24):7743-7750.

20. Poggi A, Rubartelli A, Moretta L, Zocchi MR: Expression and function of NKRP1A molecule on human monocytes and dendritic cells. Eur J Immunol 1997, 27(11):2965-2970.

21. Schroder K, Zhou R, Tschopp J: The NLRP3 Inflammasome: A Sensor for Metabolic Danger? Science 2010, 327(5963):296-300.

22. Spyrou C, Stark R, Lynch AG, Tavare S: BayesPeak: Bayesian analysis of ChIP-seq data. BMC Bioinformatics 2009, 10:299.

23. Cairns J, Spyrou C, Stark R, Smith ML, Lynch AG, Tavaré S: BayesPeak-an R package for analysing ChIP-seq data. Bioinformatics 2011, 27(5):713-714.
24. Xiao L, Rao JN, Zou T, Liu L, Marasa BS, Chen J, Turner DJ, Passaniti A, Wang $J Y$ : Induced JunD in intestinal epithelial cells represses CDK4 transcription through its proximal promoter region following polyamine depletion. Biochem J 2007, 403(3):573-581.

25. Toualbi-Abed K, Daniel F, Güller MC, Legrand A, Mauriz J-L, Mauviel A, Bernuau D: Jun $D$ cooperates with $p 65$ to activate the proximal $\mathrm{kB}$ site of the cyclin D1 promoter: role of PI3K/PDK-1. Carcinogenesis 2008, 29(3):536-543.

26. Kuhlmann A-S, Villaudy J, Gazzolo L, Castellazzi M, Mesnard J-M, Duc Dodon M: HTLV-1 HBZ cooperates with JunD to enhance transcription of the human telomerase reverse transcriptase gene (hTERT). Retrovirology 2007, 4(1):92.

27. Birney E, Stamatoyannopoulos JA, Dutta A, Guigo R, Gingeras TR, Margulies EH, Weng Z, Snyder M, Dermitzakis ET, Thurman RE, et al: Identification and analysis of functional elements in $1 \%$ of the human genome by the ENCODE pilot project. Nature 2007, 447(7146):799-816.

28. Myers RM, Stamatoyannopoulos J, Snyder M, Dunham I, Hardison RC, Bernstein BE, Gingeras TR, Kent WJ, Birney E, Wold B, et al: A user's guide to the encyclopedia of DNA elements (ENCODE). PLOS Biol 2011, 9(4):e1001046.

29. Gerstein MB, Kundaje A, Hariharan M, Landt SG, Yan K-K, Cheng C, Mu XJ, Khurana E, Rozowsky J, Alexander R, et al: Architecture of the human regulatory network derived from ENCODE data. Nature 2012, 489(7414):91-100.

30. Farnham PJ: Insights from genomic profiling of transcription factors. Nat Rev Genet 2009, 10:605-616.

31. Ghisletti S, Barozzi I, Mietton F, Polletti S, De Santa F, Venturini E, Gregory L, Lonie L, Chew A, Wei CL, et al: Identification and characterization of enhancers controlling the inflammatory gene expression program in macrophages. Immunity 2010, 32(3):317-328.

32. Barish GD, Yu RT, Karunasiri M, Ocampo CB, Dixon J, Benner C, Dent AL, Tangirala RK, Evans RM: Bcl-6 and NF-kappaB cistromes mediate opposing regulation of the innate immune response. Genes Dev 2010, 24(24):2760-2765.

33. Cao Z, Sun X, Icli B, Wara AK, Feinberg MW: Role of Krüppel-like factors in leukocyte development, function, and disease. Blood 2010, 116(22):4404-4414

34. McConnell BB, Yang W: Mammalian Krüppel-Like Factors in Health and Diseases. Physiol Rev 2010, 90(4):1337-1381.

35. Feinberg MW, Cao Z, Wara AK, Lebedeva MA, SenBanerjee S, Jain MK: Kruppel-like Factor 4 Is a Mediator of Proinflammatory Signaling in Macrophages. J Biol Chem 2005, 280(46):38247-38258.

36. Russell L, Garrett-Sinha LA: Transcription factor Ets-1 in cytokine and chemokine gene regulation. Cytokine 2010, 51(3):217-226.

37. Kamme F, Wieloch T: Induction of junD mRNA after transient forebrain ischemia in the rat. Effect of hypothermia. Brain Res Mol Brain Res 1996 43(1-2):51-56

38. Lidwell K, Griffiths R: Possible role for the FosB/JunD AP-1 transcription factor complex in glutamate-mediated excitotoxicity in cultured cerebellar granule cells. J Neurosci Res 2000, 62(3):427-439.

39. Hamanaka K, Jian M-Y, Townsley MI, King JA, Liedtke W, Weber DS, Eyal FG, Clapp MM, Parker JC: TRPV4 channels augment macrophage activation and ventilator-induced lung injury. Am J Physiol Lung Cell Mol Physiol 2010, 299(3):L353-L362.

40. Chen X, Abair TD, Ibanez MR, Su Y, Frey MR, Dise RS, Polk DB, Singh AB, Harris RC, Zent R, et al: Integrin a1 $\beta 1$ Controls Reactive Oxygen Species Synthesis by Negatively Regulating Epidermal Growth Factor ReceptorMediated Rac Activation. Mol Cell Biol 2007, 27(9):3313-3326.

41. Yi F, Xia M, Li N, Zhang C, Tang L, Li P-L: Contribution of Guanine Nucleotide Exchange Factor Vav2 to Hyperhomocysteinemic Glomerulosclerosis in Rats. Hypertension 2009, 53(1):90-96.

42. Miletic AV, Graham DB, Montgrain V, Fujikawa K, Kloeppel T, Brim K, Weaver B, Schreiber R, Xavier R, Swat W: Vav proteins control MyD88-dependent oxidative burst. Blood 2007, 109(8):3360-3368.

43. Bogunovic B, Stojakovic M, Chen L, Maric M: An Unexpected Functional Link between Lysosomal Thiol Reductase and Mitochondrial Manganese Superoxide Dismutase. J Biol Chem 2008, 283(14):8855-8862.

44. Iskander K, Li J, Han S, Zheng B, Jaiswal AK: NQO1 and NQO2 Regulation of Humoral Immunity and Autoimmunity. J Biol Chem 2006, 281(41):30917-30924.

45. Schuchardt M, Prufer J, Prufer N, Wiedon A, Huang T, Chebli M, Jankowski V, Jankowski J, Schafer-Korting M, Zidek W, et al: The endothelium-derived 
contracting factor uridine adenosine tetraphosphate induces P2Y(2)mediated pro-inflammatory signaling by monocyte chemoattractant protein-1 formation. J Mol Med 2011, 89(8):799-810.

46. Hoogeboom D, Burgering BMT: Should I stay or should I go: $\beta$-catenin decides under stress. Biochimica et Biophysica Acta (BBA) - Reviews on Cancer 2009, 1796(2):63-74.

47. Hagenbuchner J, Kuznetsov A, Hermann M, Hausott B, Obexer P, Ausserlechner MJ: FOXO3-induced reactive oxygen species are regulated by BCL2L11 (Bim) and SESN3. J Cell Sci 2012, 125(5):1191-1203.

48. Bolstad BM, Irizarry RA, Åstrand M, Speed TP: A comparison of normalization methods for high density oligonucleotide array data based on variance and bias. Bioinformatics 2003, 19(2):185-193.

49. Irizarry RA, Hobbs B, Collin F, Beazer-Barclay YD, Antonellis KJ, Scherf U, Speed TP: Exploration, normalization, and summaries of high density oligonucleotide array probe level data. Biostatistics 2003, 4(2):249-264.

50. Dai M, Wang P, Boyd AD, Kostov G, Athey B, Jones EG, Bunney WE, Myers RM, Speed TP, Akil H, et al: Evolving gene/transcript definitions significantly alter the interpretation of GeneChip data. Nucleic Acids Res 2005, 33(20):e175.

51. Lacotte S, Brun S, Muller S, Dumortier H: CXCR3, Inflammation, and Autoimmune Diseases. Ann N Y Acad Sci 2009, 1173(1):310-317.

52. Saeed Al, Bhagabati NK, Braisted JC, Liang W, Sharov V, Howe EA, Li J, Thiagarajan M, White JA, Quackenbush J: TM4 microarray software suite. Methods Enzymol 2006, 411:134-193.

53. Saeed Al, Sharov V, White J, Li J, Liang W, Bhagabati N, Braisted J, Klapa M, Currier T, Thiagarajan M, et al: TM4: a free, open-source system for microarray data management and analysis. Biotechniques 2003, 34(2):374-378

54. Huang DW, Sherman BT, Lempicki RA: Bioinformatics enrichment tools: paths toward the comprehensive functional analysis of large gene lists. Nucleic Acids Res 2009, 37(1):1-13.

55. Huang DW, Sherman BT, Lempicki RA: Systematic and integrative analysis of large gene lists using DAVID bioinformatics resources. Nat Protocols 2009, 4(1):44-57.

56. Maratou K, Behmoaras J, Fewings C, Srivastava P, D'Souza Z, Smith J, Game L, Cook T, Aitman T: Characterization of the macrophage transcriptome in glomerulonephritis-susceptible and -resistant rat strains. Genes Immun 2010, 12(2):78-89.

57. Li H, Durbin R: Fast and accurate short read alignment with BurrowsWheeler transform. Bioinformatics 2009, 25(14):1754-1760.

58. Cesaroni M, Cittaro D, Brozzi A, Pelicci PG, Luzi L: CARPET: a web-based package for the analysis of ChIP-chip and expression tiling data. Bioinformatics 2008, 24(24):2918-2920.

59. Heinz S, Benner C, Spann N, Bertolino E, Lin YC, Laslo P, Cheng JX, Murre C, Singh $\mathrm{H}$, Glass CK: Simple Combinations of Lineage-Determining Transcription Factors Prime cis-Regulatory Elements Required for Macrophage and B Cell Identities. Mol Cell 2010, 38(4):576-589.

60. Robinson JT, Thorvaldsdottir H, Winckler W, Guttman M, Lander ES, Getz G, Mesirov JP: Integrative genomics viewer. Nat Biotechnol 2011, 29(1):24-26.

doi:10.1186/1471-2164-14-92

Cite this article as: Hull et al:: Combined ChIP-Seq and transcriptome analysis identifies AP-1/JunD as a primary regulator of oxidative stress and IL-1 $\beta$ synthesis in macrophages. BMC Genomics 2013 14:92.

\section{Submit your next manuscript to BioMed Central and take full advantage of:}

- Convenient online submission

- Thorough peer review

- No space constraints or color figure charges

- Immediate publication on acceptance

- Inclusion in PubMed, CAS, Scopus and Google Scholar

- Research which is freely available for redistribution 